\title{
Fully Automatic 3D/2D Subtracted Angiography Registration
}

\author{
E. Kerrien ${ }^{1,2}$, M-O. Berger ${ }^{1}$, E. Maurincomme ${ }^{2}$, L. Launay ${ }^{2}$, R. Vaillant ${ }^{2}$, and \\ L. Picard ${ }^{3}$ \\ 1 LORIA, BP 239, 54506 Vandoeuvre lès Nancy Cedex, France \\ 2 General Electric Medical Systems Europe, BP 34, 78533 Buc Cedex, France \\ 3 Therapeutic and diagnostic neuroradiology department, CHU \\ Hôpital neurologique \\ 29, av. du Mal de Lattre de Tassigny, 54035 Nancy, Cedex, France
}

\begin{abstract}
Today, 3-D angiography volumes are routinely generated from rotational angiography sequences. In previous work [7], we have studied the precision reached by registering such volumes with classical 2-D angiography images, inferring this matching only from the sensors of the angiography machine. The error led by such a registration can be described as a 3-D rigid motion composed of a large translation and a small rotation.

This paper describes the strategy we followed to correct this error. The angiography image is compared in a two-step process to the Maximum Intensity Projection (MIP) of the angiography volume. The first step provides most of the translation by maximizing the cross-correlation. The second step recovers the residual rigid-body motion, thanks to a modified optical flow technique. A fine analysis of the equations encountered in both steps allows for a speed-up of the calculations.

This algorithm was validated on 17 images of a phantom, and 5 patients. The residual error was determined by manually indicating points of interest and was found to be around $1 \mathrm{~mm}$.
\end{abstract}

\section{Introduction}

In interventional neuroradiology, it is very important for the neuroradiologist to know, at any time, where the catheter lies within the patient's body, with a millimetric precision. This information is deduced from Digital Subtracted Angiography (DSA) images that he/she mentally links to pre-operative Magnetic Resonance (MR) images, for example, thanks to his/her anatomical knowledge.

DSA images are the cornerstone of interventional neuroradiology. Lately, neuroradiologists have been using in clinical routine 3D X-ray angiography images of the brain vasculature (3DXA). They have been proven to bring an actual supplementary help to the physicians [2]. As a consequence, the registration of DSA images with 3DXA volumes seems an extremely promising feature.

A registration between DSA images and a 3D modality is generally performed using a stereotactic frame [13]. Nevertheless, many studies have been proposing 
alternative solutions. The closest subject to our work deals with DSA/MR angiography (MRA) registration. Previous works made either use of external markers [11] and/or extract the skeletons of the vessels in both modalities [1,3,9], and matches the projection of the $3 \mathrm{D}$ skeleton with this extracted from the DSA image using various optimization schemes.

Widening our field of view, $3 \mathrm{D} / 2 \mathrm{D}$ registration has also been used to register non-subtracted angiograms to CT acquisitions. Here again, salient features are segmented: crest lines [8], contours [4] for the images and the bone surface for the volume. The registration is reached when the surface is maximally tangent to the $2 \mathrm{D}$ features. However, similarity measures, very popular in $3 \mathrm{D} / 3 \mathrm{D}$ matching studies (see [10] for a recent and complete review of research concerning medical image registration), recently appeared as a powerful alternative to the above segmentation-based methods [12].

\section{Method}

\subsection{Definition of the Problem}

We showed in a previous work [7] that this registration problem could be formalized as follows:

- estimation of a distortion field in the DSA image: we described a calibration procedure of the angiography machine to solve that first aspect;

- estimation of a conic projection matrix, similar to those used to represent cameras in computer vision: we also showed that the intrinsic parameters of this matrix could be retrieved after a simple calibration of the angiography machine. Concerning the extrinsics, our study concluded on a good estimation of the rotation part, but quite a large imprecision on the translation.

The angiography machine, once it has been calibrated, provides an initial registration which differs from the expected matching ("perfect registration") by a rigid transformation (rotation+translation) in the 3D space. The residual rotation is small, whereas the translation can be considered as unknown.

\subsection{Strategy}

The type of images to be registered is important. Our algorithm is based on the comparison of a DSA image to a conic Maximum Intensity Projection of the 3DXA volume (MIP image). Both images are $512 \times 512$. Our strategy corresponds to the problem, as described above. First, the translation is retrieved while considering that there is no error made on the rotation. The optimal position is reached once the normalized centered cross-correlation between the DSA image and the MIP image is maximal.

This first step allows the assumption that the residual error is due to a small positioning error of the 3DXA volume in the proper space of the camera (corresponding to the projection matrix defining the registration). 
In the second step, we consider that the MIP image (resulting from the first step) and the DSA image correspond to images of the same moving object, taken at times $t$ and $t^{\prime}$ respectively. Then, observing the apparent motion between $t$ and $t^{\prime}$ can give precious information about the small rigid-body transformation we are seeking. This information is extracted using a modified optical flow technique.

\section{Algorithm}

\subsection{Maximization of the Cross-Correlation}

The normalized centered cross-correlation between the DSA image, $I_{d}$, and the MIP image, $I_{m}$, is defined as:

$$
\gamma=\frac{\sum_{(x, y) \in \mathcal{D}}\left(I_{d}(x, y)-\overline{I_{d}}\right)\left(I_{m}(x, y)-\overline{I_{m}}\right)}{\sqrt{\sum_{(x, y) \in \mathcal{D}}\left(I_{d}(x, y)-\overline{I_{d}}\right)^{2} \sum_{(x, y) \in \mathcal{D}}\left(I_{m}(x, y)-\overline{I_{m}}\right)^{2}}}
$$

where $\mathcal{D}$ is the common domain to both images and $\overline{I_{d}}, \overline{I_{m}}$ are the mean of images $I_{d}$ and $I_{m}$ respectively.

Let's consider that the projection matrix $\mathbf{M}$ represents the initial registration. The final matrix $\mathbf{M}^{\prime}$ differs from $\mathbf{M}$ by the translation $\boldsymbol{D}=\left(D_{x}, D_{y}, D_{z}\right)$. Let's now consider a point $P$ which projects on pixel $(u, v)$ in the initial image (obtained by projecting the volume along $\mathbf{M}$ ) and on pixel $\left(u^{\prime}, v^{\prime}\right)$ in the final image (obtained after translation). We have:

$$
\mathbf{M}^{\prime} P=\left(s^{\prime} u^{\prime}, s^{\prime} v^{\prime}, s^{\prime}\right)^{t}=\mathbf{M} P+\boldsymbol{D}=\left(s u+D_{x}, s v+D_{y}, s+D_{z}\right)^{t}
$$

The initial matrix $\mathbf{M}$ is normalized thanks to Toscani's method [15]. We deduce:

$$
\left\{\begin{array}{l}
u^{\prime}=\frac{s}{s+D_{z}} u+\frac{D_{x}}{s+D_{z}} \\
v^{\prime}=\frac{s}{s+D_{z}} v+\frac{D_{y}}{s+D_{z}}
\end{array}\right.
$$

This formula allows for an interpretation of vector $\boldsymbol{D}: D_{x}$ and $D_{y}$ involve a translation of the initial image, while $D_{z}$ implies a zoom with respect to the upper left center of the image. As a result, this zoom entails a translation of the structures in the image. This dependency upon the parameters perturbs the optimization.

Now let's consider a movement composed of a translation $(d u, d v)$ parallel to the image plane and a zoom $G$ with respect to the center of the image $\left(u_{c}, v_{c}\right)$ :

$$
\left\{\begin{array}{l}
u^{\prime}=G u+G d u+(1-G) u_{c} \\
v^{\prime}=G v+G d v+(1-G) v_{c}
\end{array}\right.
$$

These more intuitive, independent, parameters $d u, d v$ and $G$ better fit the optimization of cross-correlation; but they are not equivalent to vector $\boldsymbol{D}$. Indeed, in equation (2), $s$ depends on the coordinates $X, Y$ and $Z$ of point $P$. Nevertheless, 
we assumed that $s$ was constant over a volume, that is a 3DXA volume could be considered as reduced to a point. A 3DXA volume roughly occupies the volume of a sphere with a diameter of $15 \mathrm{~cm}$. It is approximately located at half-distance between the focal spot of the camera and the image plane, within a system with a focal distance of approximately 1 meter. The approximation may seem rough but the matrices we obtain lead to a variation on $s$ of about $1 \%$ over the whole 3DXA volume. This validates the hypothesis and we can determine a bijection between $\boldsymbol{D}$ and $(d u, d v, G)$ by identification of (2) and (3).

The optimization procedure with the parameters $d u, d v$ and $G$ is as follows:

- exhaustive search at low resolution $(64 \times 64$ pixels). The boundaries for the variation of the parameters are either constrained by the angiography machine characteristics $(G)$ or satisfy a reasonable criterion: the images must overlap on at least one quarter of their surface. This resolution allows to keep the main arteries (diameter above $2 \mathrm{~mm}$ ).

- pseudo-exhaustive search at maximal resolution. This technique has been described by Studholme et al. [14]. Lastly, we separate the optimization over $(d u, d v)$ and $G$ : $G$ 's influence on the cost function is so small compared to $d u$ 's and $d v$ 's that the optimization will not change its initial value.

\subsection{Modified Optical Flow}

Let's now assume we found the parameters $(d u, d v, G)$ which maximize the crosscorrelation between the DSA image and the MIP image. Consequently, the initial matrix $\mathbf{M}$ is modified (see equation (1)) and the 3DXA volume is projected in order to generate a new MIP image. This image is close enough to the DSA image so that the velocity field may be computed using optical flow techniques.

Let's consider a point $P=(X, Y, Z)$ at time $t$ and the point $P^{\prime}=\left(X^{\prime}, Y^{\prime}, Z^{\prime}\right)$ reached at time $t^{\prime}$ by $P$ after a small rigid displacement, composed of a rotation $\mathbf{R}=R_{A} R_{B} R_{C}(A, B$ and $C$ are the rotation angles around the three basic vectors of the $3 \mathrm{D}$ space) and the translation $\boldsymbol{T}=(U, V, W)$ :

$$
P^{\prime}=\mathbf{R} P+\mathbf{T}
$$

That is, under the hypothesis that the rigid-body motion is small:

$$
P^{\prime}-P=\dot{P}=\Omega \times P+\boldsymbol{T} \quad \text { with } \boldsymbol{\Omega}=(A, B, C)
$$

Where the ${ }^{\cdot}$ (dot) operator is the partial differentiation with respect to time $\frac{\partial}{\partial t}$. In the proper space of the camera, the point $P$ projects on pixel $(u, v)$,

$$
u=\alpha X / Z \quad \text { and } \quad v=\alpha Y / Z
$$

where $\alpha$ stands for the ratio between the focal distance and the pixel size. From the derivation of (5) with respect to time, and combining with (4), it follows:

$$
\left\{\begin{array}{l}
\dot{u}=\alpha B-C v-\frac{A}{\alpha} u v+\frac{B}{\alpha} u^{2}+\frac{\alpha U-W u}{Z} \\
\dot{v}=-\alpha A+C u+\frac{B}{\alpha} u v-\frac{A}{\alpha} v^{2}+\frac{\alpha V-W v}{Z}
\end{array}\right.
$$


This equation defines a transformation in the image plane, but for the $1 / Z$ term. This is the classical problem one encounters who tries to retrieve the $3 \mathrm{D}$ motion from the apparent motion [5]: only 5 parameters out of 6 can be retrieved because of the undetermination on the depth of the object (given by $Z$ ) in the proper space of the camera. However, the MIP projection associates each pixel to one unique voxel. As a result, for each pixel, $Z$ is uniquely set to the depth of the corresponding voxel. Thus, equation (6) completely relates the 6 motion parameters to the pixel coordinates.

Lastly, $\dot{u}$ and $\dot{v}$ remain to be dealt with. We follow the optical flow hypothesis, which assumes that the image intensity remains constant over time [6]:

$$
\frac{d I}{d t}=\nabla I \bullet\left(\begin{array}{c}
\dot{u} \\
\dot{v}
\end{array}\right)+\dot{I}=0
$$

This is not valid when comparing the DSA image to the MIP image: both images are obtained through very different means and, moreover, do not represent the same object (real object with regard to the result of a tomographic reconstruction). However, a mere normalization of both images provides images which are similar enough to satisfy this constraint.

Combining equation (6) ( $Z$ is set for each pixel as indicated above) and equation (7), we obtain for each pixel, two equations that the 6 motion parameters must obey. This leads us to an overdetermined system of equations with 6 unknowns, which is solved using a least squares technique (pseudo-inverse).

\section{$3.3 \quad$ Speed-Up}

The two phases described above demand a large number of MIP projections. This is obvious in the optimization of the cross-correlation. Concerning the modified optical flow, results suffer from a long known disease: they are qualitatively good (we head towards the right direction) but quantitatively poor (the motion amplitude is underestimated). As a consequence, an iterative resolution of the residual motion through this technique was implemented: at the end of each iteration, the projection matrix is updated to take the newly found rigid-body transformation into account and the volume is reprojected ; this new MIP image is compared to the DSA image in the next iteration using equation (6). Therefore, we also need a large number of projections: one per iteration.

Despite all our efforts, the generation of a MIP conic projection takes 1 second (3DXA volumes are $512^{3}$ ). This constitutes a big handicap for our method, since it leads to an unacceptable calculation time: the exhaustive search at low resolution covers 32 values for $d u$ and $d v$, and 35 values for $G$. We must therefore perform $32 \times 32 \times 35=35840$ cross-correlation calculations, and, as a consequence, as many MIP projections. Given the time of 1 second per projection, this should take approximately 10 hours!

A finer analysis of the two basic equations (3) and (6) for our method, shows that they each define a 2D transformation in the image plane, if we know the motion parameters ( $d u, d v$ and $G$ for (3) and $A, B, C, U, V$ and $W$ for (6)). 
Thus, given a motion (either translation or small rigid motion), the new MIP image can be deduced from the old one with no need for an actual reprojection. This dramatically improves the calculation time.

\section{Results}

This algorithm allows for the implementation of a fully automatic 3D/2D angiography images registration in less than 90 seconds per DSA image (on a Sun UltraSparc workstation).

The gold standard for registration is usually provided by the use of a stereotactic frame. In our case, however, there is no established method to detect such a frame in 3DXA images: since the volume is reconstructed from subtracted images, the frame markers are not reconstructed The problem still remains with external markers.

Therefore, we adopted two ways of validating the result of a registration. First, the registration is visually assessed by comparing the original DSA image and the final MIP image. It allows us to state whether or not the registration succeeded, the more important information at this stage being whether or not the registration is usable for a neuroradiology intervention. Second, the error is manually estimated: given a biplane DSA sequence, each plane being registered with a 3DXA volume, we can point at salient features in the biplane images (bifurcations, marked curves, etc..) so as to reconstruct a point in the 3DXA volume. The error is the $3 \mathrm{D}$ distance between the reconstructed point and the effective location of the selected feature.

The algorithm has been tested on 17 images of a phantom and 5 biplane sequences of patients (that is, 10 DSA images). It always succeeded. The maximum 3D estimated error was $1.5 \mathrm{~mm}$ for the phantom images. However, the reconstructions of the phantom were all artifacted, lowering the quality of the registration.

Concerning the patient images, the maximum error found was $1 \mathrm{~mm}$. We display on figure 1 two examples of registration performed with our algorithm (one per line): the first line shows an internal carotid artery with a previously treated aneurysm, the second line concerns an Arterio-Veinous Malformation (AVM) fed by the vertebral artery. The third line gives zoomed versions in order to better appreciate the quality of the registration.

\section{Discussion}

A strong filiation exists with studies on frameless DSA/MRA registration. External markers are difficult to design for angiograms. Furthermore, they have not proven to be accurate enough. Masutani [11] corrects such a registration by constraining the catheter to remain on the vessels skeleton extracted in MRA. All other methods $[1,3,9]$ extract the vessels skeletons in both modality (DSA and MRA). The registration is considered to be attained when the projection of the $3 \mathrm{D}$ skeleton maximally superimposes the $2 \mathrm{D}$ skeleton. 




Fig. 1. Two examples of registration (a,b). Each line depicts a result on a DSA acquisition with 3 images: initial MIP image, MIP image at registration, DSA image. The third line shows a zoom of these results: (c1) left: DSA image, right: MIP image for example (a), (c2) same for (b). See the text for discussion of these examples.

All these skeleton-based methods have the same drawbacks. Liu [9] underlines that the projection of the centerline of the vessels in 3D does not correspond to the centerline in 2D. These techniques also generally require a test to reject some parts of the skeletons (parts which were detected in only one modality). In our opinion, the fundamental problems reside in the skeleton extraction itself.

Examples from figure 1 underline these difficulties. The image on figure 1a was acquired with a big zoom and a strong collimation (compare the DSA and MIP images). As a consequence, a lot of bifurcations, vessel superimpositions and tangencies are present (see zoom on figure 1c1) which is known to be a handicap for most segmentation methods. On the second example (figure $1 \mathrm{~b}$ and $1 \mathrm{c} 2$ ), the AVM blush would clearly perturb the skeleton extraction, though it is the region of interest where we are looking for the best registration. Thus, our technique is well suited to the $3 \mathrm{D} / 2 \mathrm{D}$ registration of complex vascular structures. 


\section{Conclusion}

We presented in this paper a new algorithm which leads to a fully automatic $3 \mathrm{D} / 2 \mathrm{D}$ subtracted angiography images registration. The initial conditions state that the DSA images are distortion-free, the intrinsics of the projection matrix are known and that a good initial guess can be made on the rotation: all of which are valid once the angiography machine has been properly calibrated [7].

\section{References}

1. Alperin N., Levin D., and Pelizzari C. "Retrospective registration of X-ray angiograms with MR images by using vessels as intrinsic landmarks" In Journal of Magnetic Resonance Imaging, volume 4, pages 139-144, 1994. 665, 669

2. Anxionnat R., Bracard S., Macho J., Da Costa E., Vaillant R., Launay L., Trousset Y., Roméas R. and Picard L. "3D angiography: clinical interest and first applications in interventional radiology" Journal of Neuroradiology, vol. 25, pages 251-262, 1998. 664

3. Feldmar J., Malandain G., and Ayache N., Fernández-Vidal S. and Maurincomme E. "Matching a 3D MR angiography volume image and 2D X-Ray angiograms" In First International Joint Conference on Computer Vision, Virtual Reality, and Robotics in Medicine and Medical Robotics and Computer Assisted Surgery, volume 1205, pages 129-140, 1997. 665, 669

4. Guéziec A., Kazanzides P., Williamson B., and Taylor R. H. "Anatomy-based registration of CT-scan and intraoperative X-ray images for guiding a surgical robot" In IEEE Transactions on Medical Imaging, volume 17, number 5, pages 715728, 1998. 665

5. Gupta N. C., Kanal L. N. "3D motion estimation from motion field" In Artificial Intelligence, volume 78, pages 45-86, 1995. 668

6. Horn B. K. P., Schunck B. G. "Determining optical flow" In Artificial Intelligence, volume 17, pages 185-203, 1981. 668

7. Kerrien E., Vaillant R., Launay L., Berger M-O., Maurincomme E. and Picard L. "Machine precision assessment for 3D/2D digital subtracted angiography images registration" In SPIE Medical Imaging, volume 3338, pages 39-49, 1998. 664, 665, 671

8. Lavallée S., Szeliski R., and Burnie L. "Matching 3D Smooth Surfaces with their 2D projections Using 3D Distance Maps" In SPIE Geometric Methods in Computer Vision, volume 1570, pages 322-336, 1991. 665

9. Liu A., Bullitt E., Pizer S. M. "3D/2D registration via skeletal near projective invariance in tubular objects" In Proceedings of MICCAI'98, W.M. Wells, A. Colchester and S. Delp Eds, Lecture Notes in Computer Science, number 1496, pages 952963, 1998. 665, 669, 670

10. Maintz J.B.A and Viergever M.A "A survey of medical image registration" In Medical Image Analysis, volume 2, number 1, pages 1-36, 1998. 665

11. Masutani Y., Furukawa C., Sonderegger M., Masamune K., Suzuki M., Dohi T., Yamane F., Iseki H., and Takakura K. "3D position visualization of catheter tip for intravascular neurosurgery using 3D structural description of vasculature" In Computer Assisted Radiology, pages 821-826, 1996. 665, 669

12. Penney G. P., Weese J., Little J. A., Hill D. L. G, and Hawkes D. J. "A comparison of similarity measures for use in 2D-3D medical image registration" In IEEE Transactions on Medical Imaging, volume 17, number 4, pages 586-595, 1998. 665 
13. Picard L., Maurincomme E., Söderman M., Feldmar J., Anxionnat R., Launay L., Ericson K., Malandain G., Bracard S., Kerrien E., Flodmark O. and Ayache N. "X-Ray angiography in stereotactic conditions: techniques and interest for interventional neuroradiology" In Stereotactic and Functional Neurosurgery, volume 68, pages 117-120, 1997. 664

14. Studholme C., Hill D., and Hawkes D. "Automated 3D registration of MR and CT images of the head" In Medical Image Analysis, volume 1, issue 2, March 1996. 667

15. Toscani, G. "Systèmes de calibration et perception du mouvement en vision artificielle" PhD thesis, Université de Paris-Sud, Orsay, 1987. 666 